\section{Distúrbios musculoesqueléticos \\ em trabalhadores do setor saúde \\ de Belo Horizonte, Minas Gerais, Brasil}

\author{
Musculoskeletal disorders among \\ healthcare workers in Belo Horizonte, \\ Minas Gerais State, Brazil
}

Rose Elizabeth Cabral Barbosa 1

Ada Ávila Assunção 1

Tânia Maria de Araújo 2
1 Universidade Federal de Minas Gerais, Belo Horizonte, Brasil.

2 Universidade Estadual de Feira de Santana, Feira de Santana, Brasil.

Correspondência R. E. C. Barbosa Universidade Federal de Minas Gerais.

Av. Professor Alfredo Balena 190, sala 733, Belo Horizonte, MG 30130-100, Brasil. rosebarbosa@ig.com.br

\section{Abstract}

This cross-sectional study investigated the prevalence of musculoskeletal disorders and associated factors in a sample of 1,808 workers (from a total of 13,602) in the municipal health system in Belo Horizonte, Minas Gerais State, Brazil. Prevalence was calculated according to self-reported pain in the upper or lower limbs and/or back, and size of associations was estimated by univariate $(p \leq 0.20)$ and multivariate Poisson regression ( $p \leq 0.05$ ). Musculoskeletal disorders showed a prevalence of $49.9 \%$ and were statistically associated with female gender, living with a partner, physical activity less than twice a week, self-reported common mental disorder, certain job positions (dentists, dental technicians, and community health workers), high physical demand, and inadequate working conditions. The results confirm the complexity of musculoskeletal disorders and suggest areas for development of health promotion programs in health services.

Cumulative Trauma Disorders; Health Occupations; Occupational Health; Working Conditions

\section{Introdução}

Trabalhadores da saúde são todos aqueles que se inserem direta ou indiretamente na prestação de serviços de saúde, no interior dos estabelecimentos de saúde ou em atividades de saúde, podendo deter ou não formação específica para o desempenho de funções atinentes à área. O principal critério adotado na definição de quem são os trabalhadores da saúde é o vínculo de trabalho no setor, independente da formação do indivíduo ${ }^{1}$.

No Brasil, em 2007, foram registrados 2.566.694 empregos no setor saúde 2 . A gama de profissionais contempla aqueles responsáveis pela atividade-fim e os inseridos no âmbito da gestão, do desenvolvimento e da manutenção dos estabelecimentos de saúde. Essa diversidade impõe análises complexas, as quais são intensificadas pela constatação dos diferenciais socioeconômicos quando comparados os diferentes grupos ocupacionais do setor.

A ocupação se constitui um construto operacional básico para a identificação dos indivíduos e suas famílias na pirâmide social. O reconhecimento da inserção social dos indivíduos na sociedade com a posição por eles desempenhadas no mercado de trabalho marca estudos de diferentes linhagens. Os trabalhadores da saúde estão envolvidos na problemática das desigualdades em saúde porque estão mais ou menos vulneráveis, a depender de sua inserção ocupacional e sua situação socioeconômica 3 . 
No campo de estudo das relações saúde e trabalho está proposto o modelo explicativo das iniquidades em saúde musculoesquelética 4,5 , pois estão suficientemente descritas as desigualdades sociais no tocante à exposição aos fatores de risco conhecidos e aos seus efeitos mórbidos. Os distúrbios musculoesqueléticos são responsáveis por elevados índices de absenteísmo e incapacidade temporária ou permanente, com reflexos negativos também para a qualidade dos serviços de saúde prestados 6 .

É reconhecido que a dor, principal sintoma dos distúrbios musculoesqueléticos, tem origem multifatorial e é prevalente entre os trabalhadores da saúde. Fatores biomecânicos, como levantamento de peso e manutenção de posturas forçadas e prolongadas, são de risco para a dor no dorso ${ }^{7}$. Movimentos repetitivos de membros superiores ou gestos realizados em contração estática têm sido associados à dor nesses membros e no pescoço ${ }^{8}$. Não restam dúvidas de que fatores psicossociais desencadeiam ou agravam a dor musculoesquelética ${ }^{9}$, estando também implicadas características individuais, como idade, sexo, comportamentos (tabagismo, sedentarismo, por exemplo) e comorbidades 10,11. Apesar dos múltiplos determinantes dos distúrbios musculoesqueléticos, são raros os estudos que abordam conjuntamente características individuais, do emprego, além de fatores físicos e psicossociais do trabalho.

Embora não possamos modificar as características pessoais dos trabalhadores, os elementos relacionados ao nível individual podem ser modificados, a fim de melhorar as condições de trabalho e reduzir os efeitos sobre o trabalhador. Entre eles, destacam-se as condições do emprego; a organização, o conteúdo e a demanda do trabalho; o incentivo aos hábitos e comportamentos saudáveis 5 .

O objetivo do presente estudo foi avaliar a associação entre a prevalência de distúrbios musculoesqueléticos e características individuais, do emprego e do trabalho em uma população de trabalhadores da rede municipal de saúde de Belo Horizonte, Minas Gerais, Brasil.

\section{Metodologia}

Estudo transversal, que focalizou uma amostra de trabalhadores da rede municipal de saúde de Belo Horizonte, em efetivo exercício profissional na unidade estudada. O sistema de saúde da capital encontra-se estruturado, com base na área geográfica, em nove distritos sanitários: Barreiro, Centro-Sul, Leste, Nordeste, Noroeste, Norte, Oeste, Pampulha e Venda Nova 12.
À época da coleta de dados, o setor contava com 13.602 trabalhadores. Foram considerados elegíveis todos os profissionais vinculados à rede, independentemente do vínculo empregatício (permanente, temporário, estágio). Aqueles que não se encontravam no serviço por motivo de férias, transferência, aposentadoria ou morte foram substituídos, respeitando-se a função ou cargo, nível de assistência e região.

O estudo sobre distúrbios musculoesqueléticos analisa dados colhidos no bojo de um inquérito que abordou outros desfechos. A estratégia utilizada para o cálculo amostral do referido inquérito se deteve no desfecho de interesse de menor prevalência. A posteriori, foi calculada a amostra específica para o estudo da associação entre os fatores de interesse e distúrbios musculoesqueléticos. Considerando ainda tratar-se de um estudo exploratório, no qual um conjunto de variáveis foi investigado (portanto não havia uma associação principal sob análise), foram conduzidos cálculos, com base em dados da literatura 13,14,15 e das frequências obtidas no estudo em questão, incluindo as variáveis inseridas na análise (sexo, ocupação, atividades de lazer, prática de atividades físicas, condições do ambiente de trabalho, ritmo de trabalho, transtornos mentais comuns, demanda psicológica e modelo demanda-controle, dentre outras). Tomou-se $n$ da amostra a estimativa que resultou no maior tamanho amostral (para demanda psicológica). Para o cálculo, considerando demanda psicológica, foram assumidos os seguintes parâmetros: frequência de distúrbios musculoesqueléticos em baixa demanda (não expostos) de $20,1 \%$ e em alta demanda (expostos), de 28,3\%; intervalo de 95\% de confiança (IC95\%) e poder de $90 \%$, chegando-se a um $n$ amostral de 1.191 trabalhadores.

Para a seleção dos trabalhadores da saúde a serem estudados, foi conduzida uma amostragem estratificada proporcional considerando três critérios (variáveis) de estratificação: distrito sanitário, nível de complexidade da assistência e grupo ocupacional. O acesso à lista de funcionários disponível no departamento de recursos humanos permitiu identificar a distribuição dos trabalhadores. Após a estimativa do tamanho amostral, calculou-se a composição da amostra pela participação percentual dos trabalhadores, segundo a regional ou distrito sanitário; assim, estipulouse o número de pessoas a serem estudadas em cada distrito. Em seguida, calculou-se o número a ser estudado em cada nível de complexidade da assistência (centros de saúde, especialidades, urgência e gerências distritais) também pela participação percentual dos trabalhadores segundo o nível. Finalmente, em cada nível de complexi- 
dade, definiu-se a participação percentual de cada um dos dez grupos ocupacionais constituídos conforme a classificação da Organização Mundial da Saúde (OMS) 16. Processou-se, então, o sorteio dos trabalhadores a serem estudados, com base em uma lista de números aleatórios gerada pelo Epi Info (Centers for Disease Control and Prevention, Atlanta, Estados Unidos).

Para a coleta de dados, realizada entre setembro de 2008 e janeiro de 2009, empregouse um questionário autoaplicado, previamente testado em um estudo-piloto em unidades de saúde, as quais, por essa razão, foram excluídas do sorteio. O questionário foi organizado em blocos de questões referentes a características sociodemográficas, informações gerais sobre o trabalho, características do ambiente de trabalho, fatores psicossociais do trabalho, atividades domésticas e hábitos de vida, qualidade de vida, aspectos relacionados à saúde e atos de violência-vitimização.

O preenchimento do questionário foi conduzido por uma equipe de nove entrevistadores treinados, que se dirigiu à unidade do participante após contato por telefone. Foram realizadas até três visitas aos locais de trabalho de cada entrevistado no intuito de localizar o trabalhador sorteado, considerando-se perda quando este não era encontrado na terceira tentativa.

Para a definição da variável resposta, presença de distúrbios musculoesqueléticos, considerouse o autorrelato de ocorrência de dor nos braços, nas pernas ou nas costas. A frequência da dor foi medida numa escala do tipo Likert: $1=$ nunca; $2=$ raramente; 3 = pouco frequente; 4 = frequente e 5 = muito frequente. Foi considerada presença de distúrbios musculoesqueléticos quando o trabalhador referiu a dor como "frequente" ou "muito frequente" em, pelo menos, um dos segmentos corporais analisados. Ausência de dor foi classificada quando a resposta variou de 1 a 3 (nunca, raramente ou pouco frequente).

As variáveis explicativas consideradas na análise dos dados foram as seguintes:

- Características individuais: informações sociodemográficas (sexo, idade, situação conjugal e grau de escolaridade), hábitos de vida (participação em atividades de lazer, prática de atividades físicas e tabagismo) e condições de saúde (licença médica, falta ou afastamento do trabalho por problemas de saúde nos últimos 12 meses e presença de transtorno mental comum).

- Características do emprego: cargo que ocupa, tempo de serviço público e na unidade estudada, regime de trabalho, existência de outro emprego, jornada de trabalho semanal na unidade e jornada total, renda bruta mensal relativa ao trabalho na unidade.
- Características do trabalho: relação entre as exigências das tarefas e os recursos disponíveis, condições do ambiente de trabalho, demanda física do trabalho e sobrecarga doméstica, aspectos psicossociais do trabalho (demandas emocional e psicológica do trabalho, controle sobre o trabalho e suporte social dos colegas e da chefia).

Os transtornos mentais comuns (TMC) foram avaliados de acordo com escores obtidos no Self Reporting Questionnaire (SRQ-20), um instrumento desenvolvido pela OMS para avaliar morbidade psíquica em países em desenvolvimento, que identifica possíveis casos de TMC 17. O questionário é composto por 20 questões, com respostas dicotômicas (sim ou não), incluindo sintomas emocionais e físicos associados a quadros psiquiátricos. Definiu-se o ponto de corte para classificação de suspeitos de TMC em sete ou mais respostas positivas, procedimento adotado em outros estudos 18,19.

A variável relação entre as exigências das tarefas e os recursos disponíveis originou-se de uma pergunta presente no questionário, com quatro opções de respostas: boa, regular, ruim ou muito ruim (as duas últimas alternativas foram agrupadas na mesma categoria ruim/muito ruim).

A variável condições do ambiente de trabalho expressa as características do ambiente físico em que os trabalhadores da saúde desempenham suas funções. Essa variável abarcou avaliação da ventilação, temperatura, iluminação, recursos técnicos e equipamentos (incluindo cadeiras e mesas) do local de trabalho, sendo os itens avaliados pelos respondentes em escala de 1 a 3: precários (1), razoáveis (2) ou satisfatórios (3). Avaliou-se, ainda, o ruído originado no trabalho e fora dele como desprezível (3), razoável (2), elevado e insuportável (1). Valendo-se do somatório das respostas para cada item relativo ao ambiente físico, construiu-se um escore global: quanto maior o escore, melhores eram as condições ambientais de trabalho. Para fins de análise, o escore criado foi categorizado, com base nos quartis, em: condições precárias (corte no primeiro quartil), razoáveis (corte no segundo quartil), satisfatórias (terceiro quartil) e ótimas (último quartil).

A variável demanda física do trabalho originou-se de respostas a questões sobre adoção de posturas relacionadas à dor ou desconforto, ficar de pé ou assentado durante longos períodos, realização de caminhadas, necessidade de levantar, carregar ou empurrar peso excessivo, auxílio na movimentação de pacientes e realização de pausas durante a jornada de trabalho. Cada pergunta apresentava quatro opções de resposta $(1=$ nunca, 2 = raramente, 3 = às vezes e $4=$ sempre). Com base no somatório dos itens supracitados, 
criou-se um escore de demanda física, que foi, em seguida, categorizado pelos tercis em baixa demanda (valores iguais ou abaixo do segundo tercil) e alta demanda (valores acima do segundo tercil).

Os médicos foram considerados categoria ocupacional de referência por terem nível socioeconômico geralmente reconhecido como sendo superior ao das outras ocupações em saúde. Esses profissionais permanecem, na maioria dos casos, em consultórios ou enfermarias, com poucas incursões a campo. Raramente os médicos transportam cargas ou manipulam os pacientes acamados ou com restrição física, tampouco se deslocam por longos trajetos, realidade que indicaria menor exposição às demandas físicas no desenvolvimento das atividades.

Para a mensuração da variável sobrecarga doméstica, considerou-se a realização de quatro tarefas básicas do lar: cozinhar, limpar, lavar e passar, conforme indicador proposto por Thierney et al. 20 , ponderada pelo número de potenciais beneficiários desse tipo de trabalho. A sobrecarga doméstica foi, portanto, avaliada a partir do somatório das tarefas do lar, ponderado pelo número de moradores, exceto o próprio entrevistado $\left(\sum\right.$ lavar + passar + limpar + cozinhar $)$ x (M-1) 18 . Para fins de análise, o escore de sobrecarga doméstica foi dicotomizado com base nos tercis da distribuição obtida: baixa sobrecarga (correspondeu aos valores iguais ou abaixo do segundo tercil) e alta sobrecarga doméstica (valores acima do segundo tercil).

Os aspectos psicossociais do trabalho foram avaliados por meio do Job Content Questionnaire (JCQ), instrumento desenvolvido de acordo com o modelo demanda-controle 21,22 , que privilegia duas dimensões psicossociais: o controle sobre o trabalho e a demanda psicológica advinda deste. A partir da combinação dessas duas dimensões, o modelo distingue situações de trabalho específicas que, por sua vez, estruturam riscos diferenciados à saúde 22 .

Para demanda psicológica do trabalho, foi construído um indicador resultante do somatório dos valores referentes às questões relacionadas ao ritmo de trabalho, tempo para a realização das tarefas, tarefas conflitantes e volume excessivo de trabalho. Com base na mediana, a variável foi dicotomizada: os valores iguais ou abaixo do ponto de corte foram categorizados como baixa demanda; os acima, como alta demanda.

A variável controle sobre o próprio trabalho incluiu duas dimensões, totalizando nove questões: seis para mensuração do uso de habilidades (aprendizagem de coisas novas, criatividade, desenvolvimento de habilidades especiais e possibilidade de realização de diferentes tarefas) e três para medir autoridade decisória (liberdade para decidir como realizar as tarefas e possibilidade de tomada de decisões) ${ }^{22}$. Essa variável foi construída a partir do somatório dos itens relativos a essas duas dimensões e foi dicotomizada com base na mediana: os valores iguais ou abaixo do ponto de corte foram categorizados como baixo controle; os acima, como alto controle.

Com base na combinação entre níveis de demanda e controle, estabeleceram-se os quatro grupos previstos no modelo demanda-controle: baixa exigência (combinação de baixa demanda e alto controle), trabalho passivo (baixa demanda e baixo controle), trabalho ativo (alta demanda e alto controle) e alta exigência (alta demanda e baixo controle) 21,23 .

Para a variável suporte social, foi utilizado um indicador resultante do somatório dos valores referentes às questões relacionadas ao suporte dos colegas de trabalho e da chefia. Adotou-se ponto de corte na mediana: valores iguais ou abaixo da mediana foram categorizados como baixo suporte; os acima, como alto suporte.

A variável demanda emocional do trabalho originou-se da pergunta "Meu trabalho me exige muito emocionalmente", cujas possibilidades de resposta eram: discordo fortemente e discordo (agrupadas em baixa demanda); concordo e concordo fortemente (agrupadas em alta demanda).

Os dados coletados foram analisados no software estatístico Stata 10.0 (Stata Corp., College Station, Estados Unidos). Para a análise dos fatores associados à presença de distúrbios musculoesqueléticos, utilizou-se a regressão de Poisson com estimação de variância robusta, tendo como categoria de referência a ausência de distúrbios musculoesqueléticos. Inicialmente, realizou-se análise descritiva, estimando-se as frequências relativas de acordo com as categorias das variáveis do estudo. Além disso, calculou-se a prevalência de distúrbios musculoesqueléticos no total da amostra. A magnitude das associações entre as variáveis foi estimada calculando-se as razões de prevalência (RP) e seus respectivos IC95\%. A análise univariada foi utilizada para verificar a existência de associações brutas da prevalência de distúrbios musculoesqueléticos com cada uma das categorias das variáveis explicativas. As variáveis com valor de $\mathrm{p} \leq 0,20$ foram selecionadas para entrar na análise multivariada, permanecendo no modelo final apenas os fatores associados ao nível de $\mathrm{p} \leq 0,05$.

Os questionários não identificavam os respondentes, preservando a confidencialidade dos dados obtidos. O estudo foi aprovado pelo Comitê de Ética em Pesquisa da Universidade Federal 
de Minas Gerais (parecer no. 542/07). Termo de Consentimento Livre e Esclarecido foi requerido de todos os participantes do estudo.

\section{Resultados}

Foram sorteados 2.205 trabalhadores da rede municipal de saúde de Belo Horizonte, resultando em um total de 1.808 questionários respondidos. A pergunta que deu origem ao desfecho investigado foi respondida corretamente por 1.687 sujeitos, obtendo uma taxa de resposta de $76,5 \%$ (1.687/2.205).

A prevalência de distúrbios musculoesqueléticos foi de $36,5 \%$ para o dorso, 34,3\% para os membros inferiores e $20,4 \%$ para os membros superiores. A prevalência de distúrbios musculoesqueléticos relacionada a qualquer um dos três segmentos corporais foi de 49,9\%: 21,7\% referiram dor em apenas um dos três segmentos corporais analisados; $16,1 \%$ em dois segmentos e $12,1 \%$ nos três segmentos.

A maioria da população estudada era composta por mulheres $(71,6 \%)$ e $54,4 \%$ declararam viver com um companheiro. Quanto à idade, a média foi 40,8 $\pm 11,1$ anos, variando de 16 a 73. Entre os trabalhadores da saúde estudados, $54,2 \%$ tinham o ensino médio, técnico ou superior incompleto; $53,6 \%$ estavam no serviço público há menos de dez anos; e a jornada semanal média de trabalho na unidade estudada era de $32,4 \pm 10,2$ horas. Aproximadamente 38\% relataram ter outro emprego, e a jornada semanal média total, incluindo o conjunto de empregos, era superior a 44 horas para $31,4 \%$ dos respondentes. A renda média era $\mathrm{R} \$ 1.932,12$, e a mediana, $\mathrm{R} \$ 1.000,00$.

A prevalência de distúrbios musculoesqueléticos foi maior no grupo feminino, na faixa etária entre 35 e 46 anos e entre os que viviam com companheiro. Em relação à escolaridade, no grupo dos sujeitos com distúrbios musculoesqueléticos, predominou o ensino médio, técnico ou superior incompleto (48\%), seguido de ensino superior completo ou pós-graduação $(47,4 \%)$ (Tabela 1).

Maiores prevalências de distúrbios musculoesqueléticos foram verificadas entre os trabalhadores que relataram não participar de atividades de lazer, entre os que não praticavam atividades físicas e no grupo de não fumantes (Tabela 1). As prevalências foram mais elevadas, também, entre os que faltaram ao trabalho (sem menção à causa), foram licenciados ou afastados devido a problemas de saúde nos 12 meses anteriores à pesquisa e entre aqueles com probabilidade de TMC (Tabela 1).
Foram observadas maiores prevalências de distúrbios musculoesqueléticos no grupo com maior tempo de trabalho no serviço público ( $>10$ anos) e na unidade atual (> 5 anos), assim como entre aqueles que relataram renda mensal de até R\$ 600,00 (Tabela 2). Verificaram-se maiores prevalências de distúrbios musculoesqueléticos entre os profissionais técnicos de nível superior $(61,4 \%)$, dentistas e técnicos de odontologia (61\%), agentes comunitários de saúde (ACS) $(59,6 \%)$, profissionais técnicos de nível médio (51,5\%) e enfermeiros e técnicos de enfermagem (50\%). Por outro lado, as menores prevalências foram identificadas entre os fisioterapeutas, terapeutas ocupacionais, psicólogos e afins $(30,4 \%)$ (Tabela 2$)$.

No tocante às características do trabalho, as maiores prevalências de distúrbios musculoesqueléticos foram observadas no grupo que relatou desequilíbrio entre as exigências das tarefas e os recursos disponíveis; entre os trabalhadores expostos a condições ambientais precárias, quando comparados aos respondentes expostos a ótimas condições; entre os que desempenhavam tarefas sob alta demanda física e, ainda, entre aqueles com alta sobrecarga doméstica (Tabela 3). Quanto aos aspectos psicossociais do trabalho, a prevalência de distúrbios musculoesqueléticos foi maior entre os que relataram altas demandas emocional e psicológica do trabalho, baixo controle sobre este e baixo suporte social por parte dos colegas e do chefe, como também entre os que realizavam tarefas sob alta exigência (alta demanda, baixo controle) (Tabela 3).

Os resultados da análise univariada, apresentados nas Tabelas 1, 2 e 3, mostram que a maioria das variáveis foram estatisticamente associadas à prevalência de distúrbios musculoesqueléticos ao nível de $\mathrm{p} \leq 0,20$. Apenas hábito de fumar, regime e jornada de trabalho, existência de outro emprego e renda não foram associados.

A Tabela 4 apresenta os resultados do modelo final da análise multivariada com os fatores que permaneceram associados ao nível de $\mathrm{p} \leq$ 0,05 . A maior prevalência de distúrbios musculoesqueléticos permaneceu positivamente associada a ser do sexo feminino ( $p<0,001)$; viver com um companheiro ( $\mathrm{p}<0,05)$; praticar atividade física somente uma ou duas vezes por semana $(\mathrm{p}<0,05)$ ou não praticar atividade física $(\mathrm{p}<0,01)$; apresentar relato compatível com TMC ( $\mathrm{p}<0,001)$; exercer cargos de dentista e técnico de odontologia $(\mathrm{p}<0,05)$ e ACS $(\mathrm{p}<0,01)$. No tocante às características do trabalho, a prevalência de distúrbios musculoesqueléticos associouse significativamente à alta demanda física do trabalho $(\mathrm{p}<0,01)$ e às inadequadas condições 
Prevalência de distúrbios musculoesqueléticos segundo características sociodemográficas e de estilo de vida, entre trabalhadores municipais da saúde de Belo Horizonte, Minas Gerais, Brasil.

\begin{tabular}{|c|c|c|}
\hline Variáveis & Prevalência (\%) & RP (IC95\%) \\
\hline \multicolumn{3}{|l|}{ Sexo } \\
\hline Masculino & 28,2 & 1,00 \\
\hline Feminino & 54,3 & $1,97(1,70-2,27)$ \\
\hline \multicolumn{3}{|l|}{ Idade (anos) } \\
\hline Até 34 & 44,3 & 1,00 \\
\hline $35-46$ & 49,3 & $1,17(1,03-1,31)$ \\
\hline 47 ou mais & 46,5 & $1,13(0,99-1,27)$ \\
\hline \multicolumn{3}{|l|}{ Situação conjugal } \\
\hline Sem companheiro(a) & 43,9 & 1,00 \\
\hline Com companheiro(a) & 49,0 & $1,13(1,02-1,24)$ \\
\hline \multicolumn{3}{|l|}{ Grau de escolaridade } \\
\hline Ensino Superior completo ou Pós-graduação & 47,4 & 1,00 \\
\hline Ensino Médio, Técnico ou Superior incompleto & 48,0 & $1,03(0,93-1,14)$ \\
\hline Ensino Fundamental & 36,8 & $0,81(0,64-0,99)$ \\
\hline \multicolumn{3}{|l|}{ Participação em atividades de lazer } \\
\hline Sim & 43,8 & 1,00 \\
\hline Não & 55,4 & $1,30(1,17-1,43)$ \\
\hline \multicolumn{3}{|l|}{ Prática de atividades físicas } \\
\hline Sim, 3 ou mais vezes por semana & 36,8 & 1,00 \\
\hline Sim, 1 ou 2 vezes por semana & 44,2 & $1,23(1,05-1,43)$ \\
\hline Não & 55,6 & $1,57(1,36-1,81)$ \\
\hline \multicolumn{3}{|l|}{ Fumante atual } \\
\hline Não & 47,3 & 1,00 \\
\hline Sim & 43,5 & $0,92(0,79-1,05)$ * \\
\hline \multicolumn{3}{|l|}{ Licença médica, falta ou afastamento do trabalho por } \\
\hline \multicolumn{3}{|l|}{ problemas de saúde nos últimos 12 meses } \\
\hline Não & 39,4 & 1,00 \\
\hline Sim & 55,5 & $1,39(1,26-1,53)$ \\
\hline \multicolumn{3}{|l|}{ Transtorno mental comum } \\
\hline Ausente & 39,4 & 1,00 \\
\hline Presente & 70,4 & $1,79(1,64-1,96)$ \\
\hline
\end{tabular}

IC95\%: intervalo de 95\% de confiança; RP: razão de prevalência.

${ }^{*} p>0,20$.

ambientais: precárias ( $\mathrm{p}<0,001)$, razoáveis $(\mathrm{p}<$ $0,001)$ e satisfatórias $(\mathrm{p}<0,05)$.

\section{Discussão}

O presente estudo encontrou maior prevalência de distúrbios musculoesqueléticos entre as mulheres e entre os que declararam viver com um companheiro. Não se observaram diferenças em relação à escolaridade e à renda. As taxas de distúrbios musculoesqueléticos foram maiores entre aqueles com probabilidade de TMC e com baixa ou nula frequência de atividades físicas.
Chamam a atenção as elevadas prevalências de distúrbios musculoesqueléticos entre os dentistas, técnicos de odontologia e entre os ACS. Observou-se prevalência mais elevada no grupo com pior avaliação das condições ambientais e no grupo que informou trabalhar sob demanda física elevada.

As características individuais (sexo e vida conjunta) já foram associadas à prevalência de distúrbios musculoesqueléticos em outros estudos 8,13,24,25,26,27. Sabe-se que a dupla jornada de trabalho e a restrição do tempo de recuperação são predominantes entre as mulheres, com gradiente positivo para aquelas que vivem com 
Prevalência de distúrbios musculoesqueléticos segundo características do emprego, entre trabalhadores municipais de saúde de Belo Horizonte, Minas Gerais, Brasil.

\begin{tabular}{|c|c|c|}
\hline Variáveis & Prevalência (\%) & RP (IC95\%) \\
\hline \multicolumn{3}{|l|}{ Ocupação } \\
\hline Médicos & 41,9 & 1,00 \\
\hline Enfermeiros e técnicos de enfermagem & 50,0 & $1,31(1,08-1,59)$ \\
\hline Dentistas e técnicos de odontologia & 61,0 & $1,50(1,19-1,88)$ \\
\hline Bioquímicos e técnicos de laboratório & 36,4 & $0,91(0,60-1,37)$ \\
\hline Profissionais envolvidos com a vigilância & 36,3 & $0,91(0,68-1,20)$ \\
\hline Agentes comunitários de saúde & 59,6 & $1,49(1,24-1,78)$ \\
\hline Fisioterapeutas, terapeutas ocupacionais, psicólogos e afins & 30,4 & $0,78(0,41-1,45)$ \\
\hline Profissionais técnicos de nível superior & 61,4 & $1,46(1,13-1,87)$ \\
\hline Profissionais técnicos de nível médio & 51,5 & $1,30(1,07-1,56)$ \\
\hline Administrativos, serviços gerais e outros & 37,6 & $0,93(0,76-1,13)$ \\
\hline \multicolumn{3}{|l|}{ Tempo de serviço público (anos) } \\
\hline Até 10 & 44,6 & 1,00 \\
\hline Acima de 10 & 49,9 & $1,15(1,04-1,26)$ \\
\hline \multicolumn{3}{|l|}{ Tempo de serviço na unidade atual (anos) } \\
\hline Até 5 & 43,6 & 1,00 \\
\hline Acima de 5 & 50,0 & $1,18(1,06-1,29)$ \\
\hline \multicolumn{3}{|l|}{ Regime de trabalho } \\
\hline Diurno & 47,2 & 1,00 \\
\hline Noturno & 47,3 & $1,01(0,77-1,33)$ * \\
\hline \multicolumn{3}{|l|}{ Jornada de trabalho semanal na unidade atual (horas) } \\
\hline Até 36 & 45,7 & 1,00 \\
\hline $40-44$ & 47,9 & $1,04(0,94-1,14)$ * \\
\hline \multicolumn{3}{|l|}{ Outro emprego } \\
\hline Não & 46,0 & 1,00 \\
\hline Sim & 45,5 & $0,98(0,88-1,09)$ * \\
\hline \multicolumn{3}{|l|}{ Jornada total de trabalho semanal (horas) } \\
\hline Até 36 & 42,5 & 1,00 \\
\hline $37-44$ & 46,7 & $1,09(0,94-1,26)$ * \\
\hline Acima de 44 & 46,1 & $1,06(0,90-1,24)$ * \\
\hline \multicolumn{3}{|l|}{ Renda (em Reais) } \\
\hline Até 600,00 & 50,1 & 1,00 \\
\hline $601,00-1.200,00$ & 45,5 & $0,93(0,82-1,05)$ * \\
\hline Acima de $1.200,00$ & 48,1 & $0,96(0,85-1,07)$ * \\
\hline
\end{tabular}

IC95\%: intervalo de $95 \%$ de confiança; RP: razão de prevalência.

${ }^{*} p>0,20$.

companheiro. As mulheres estariam sobrecarregadas pelas tarefas domésticas, com menos tempo para atividades de lazer, e, uma vez privadas do necessário repouso, teriam enfraquecida a recuperação dos efeitos provocados pelas tarefas ocupacionais. Nesse quadro, espera-se aumento da ocorrência de sintomas musculoesqueléticos, dos sintomas de estresse, dor e fadiga 28,29 .

Encontrou-se maior prevalência, com gradiente positivo, de sujeitos com distúrbios mus- culoesqueléticos no grupo que relatou baixa frequência ou ausência de atividade física, sendo respectivamente $19 \%$ e $27 \%$ maior nas referidas situações, se comparado ao grupo que relatou três ou mais episódios de atividades por semana. Provavelmente, a capacidade para a prática da atividade física esteja comprometida pelas limitações esperadas em sujeitos com relato de distúrbios musculoesqueléticos 30 , mas não é imprudente supor que tal resultado seja a expressão do efeito protetor das atividades físicas para um 
Prevalência de distúrbios musculoesqueléticos segundo características do trabalho, entre trabalhadores municipais da saúde de Belo Horizonte, Minas Gerais, Brasil.

\begin{tabular}{|c|c|c|}
\hline Variáveis & Prevalência (\%) & RP (IC95\%) \\
\hline \multicolumn{3}{|c|}{ Relação exigência das tarefas/recursos disponíveis } \\
\hline Boa & 37,3 & 1,00 \\
\hline Regular & 49,0 & $1,27(1,12-1,42)$ \\
\hline Ruim/Muito ruim & 61,5 & $1,60(1,40-1,83)$ \\
\hline \multicolumn{3}{|c|}{ Condições do ambiente de trabalho } \\
\hline Ótimas & 34,2 & 1,00 \\
\hline Satisfatórias & 41,5 & $1,28(1,05-1,54)$ \\
\hline Razoáveis & 50,4 & $1,52(1,25-1,83)$ \\
\hline Precárias & 57,5 & $1,72(1,43-2,06)$ \\
\hline \multicolumn{3}{|l|}{ Demanda física } \\
\hline Baixa & 40,2 & 1,00 \\
\hline Alta & 60,7 & $1,51(1,38-1,66)$ \\
\hline \multicolumn{3}{|c|}{ Sobrecarga doméstica } \\
\hline Baixa & 43,6 & 1,00 \\
\hline Alta & 57,4 & $1,35(1,22-1,50)$ \\
\hline \multicolumn{3}{|c|}{ Demanda emocional do trabalho } \\
\hline Baixa & 35,9 & 1,00 \\
\hline Alta & 50,6 & $1,41(1,23-1,60)$ \\
\hline \multicolumn{3}{|c|}{ Controle sobre o trabalho } \\
\hline Alto & 44,0 & 1,00 \\
\hline Baixo & 48,5 & $1,12(1,00-1,23)$ \\
\hline \multicolumn{3}{|c|}{ Demanda psicológica do trabalho } \\
\hline Baixa & 41,8 & 1,00 \\
\hline Alta & 52,4 & $1,22(1,10-1,35)$ \\
\hline \multicolumn{3}{|c|}{ Suporte social dos colegas e do chefe } \\
\hline Alto & 42,7 & 1,00 \\
\hline Baixo & 49,6 & $1,16(1,05-1,28)$ \\
\hline \multicolumn{3}{|c|}{ Modelo demanda-controle } \\
\hline Baixa exigência & 38,1 & 1,00 \\
\hline Trabalho passivo & 43,7 & $1,18(0,99-1,39)$ \\
\hline Trabalho ativo & 48,6 & $1,28(1,07-1,51)$ \\
\hline Alta exigência & 55,2 & $1,44(1,22-1,69)$ \\
\hline
\end{tabular}

IC95\%: intervalo de 95\% de confiança; RP: razão de prevalência.

grande leque de morbidades, incluindo aquelas do aparelho musculoesquelético ${ }^{31}$. Em todo caso, em se tratando de um estudo transversal, não é possível indicar a direção desta evidência.

A frequência de atividades físicas também pode explicar as morbidades psíquicas reveladas nos achados compatíveis com TMC, pois se sabe que exerce influência positiva sobre o sono e efeito protetor contra o estresse 32,33.

O grupo de trabalhadores com relato compatível com TMC apresentou maior prevalência de distúrbios musculoesqueléticos. Esse resultado é coerente com os de estudo anterior, que focali- zou uma amostra de dentistas, tendo evidenciado associação positiva entre distúrbios musculoesqueléticos e depressão, tensão, ansiedade e preocupação excessiva 34 .

O grupo de dentistas e técnicos de odontologia e o grupo de ACS apresentaram maior prevalência de DME quando comparados aos médicos. Chama a atenção o dado negativo entre o pessoal da enfermagem e também entre os fisioterapeutas e terapeutas ocupacionais, contrariando publicações recentes 6,35.

Quanto às prevalências encontradas entre dentistas e técnicos de odontologia, os resulta- 
Razões de prevalência (RP) de distúrbios musculoesqueléticos e respectivos intervalos de 95\% de confiança (IC95\%) para as variáveis do modelo final obtido mediante análise multivariada.

\begin{tabular}{|c|c|}
\hline Variáveis & RP (IC95\%) \\
\hline \multicolumn{2}{|l|}{ Sexo } \\
\hline Masculino & 1,00 \\
\hline Feminino & $1,48(1,25-1,73)$ * \\
\hline \multicolumn{2}{|l|}{ Situação conjugal } \\
\hline Sem companheiro(a) & 1,00 \\
\hline Com companheiro(a) & $1,14(1,02-1,27) * \star$ \\
\hline \multicolumn{2}{|l|}{ Prática de atividades físicas } \\
\hline Sim, 3 ou mais vezes por semana & 1,00 \\
\hline Sim, 1 ou 2 vezes por semana & $1,19(1,02-1,38) * *$ \\
\hline Não & $1,27(1,09-1,47) * \star \star$ \\
\hline \multicolumn{2}{|l|}{ Transtorno mental comum } \\
\hline Ausente & 1,00 \\
\hline Presente & $1,60(1,44-1,77)$ * \\
\hline \multicolumn{2}{|l|}{ Ocupação } \\
\hline Médicos & 1,00 \\
\hline Enfermeiros e técnicos de enfermagem & $1,02(0,83-1,25)$ \\
\hline Dentistas e técnicos de odontologia & $1,29(1,01-1,63) * \star$ \\
\hline Bioquímicos e técnicos de laboratório & $0,94(0,60-1,46)$ \\
\hline Profissionais envolvidos com a vigilância & $1,05(0,80-1,37)$ \\
\hline Agentes comunitários de saúde & $1,37(1,12-1,66) * \star \star$ \\
\hline Fisioterapeutas, terapeutas ocupacionais, psicólogos e afins & $0,74(0,37-1,44)$ \\
\hline Profissionais técnicos de nível superior & $1,33(0,99-1,78)$ \\
\hline Profissionais técnicos de nível médio & $1,15(0,94-1,40)$ \\
\hline Administrativos, serviços gerais e outros & $1,02(0,82-1,26)$ \\
\hline \multicolumn{2}{|l|}{ Condições do ambiente de trabalho } \\
\hline Ótimas & 1,00 \\
\hline Satisfatórias & $1,33(1,06-1,65) * *$ \\
\hline Razoáveis & $1,55(1,24-1,92)$ * \\
\hline Precárias & $1,57(1,26-1,95)$ * \\
\hline \multicolumn{2}{|l|}{ Demanda física } \\
\hline Baixa & 1,00 \\
\hline Alta & $1,27(1,13-1,41)$ * \\
\hline
\end{tabular}

${ }^{*} p<0,001$

$* * p<0,05$;

$\star \star \star * p<0,01$.

dos são convergentes aos obtidos pelas pesquisas em uma amostra de 430 dentistas na Grécia, quando $62 \%$ dos profissionais relataram pelo menos uma queixa de dor musculoesquelética 8; porém, são inferiores aos encontrados entre 285 profissionais na Austrália, estudo em que 87,2\% dos sujeitos relataram pelo menos uma queixa de dor musculoesquelética 36 . As altas prevalências encontradas entre os profissionais da odontologia podem ser explicadas pela exposição às posturas desconfortáveis durante os atendimen- tos, aos tipos de movimentos realizados, ao manejo de ferramentas vibratórias e à sobrecarga de trabalho (número de atendimentos e horas trabalhadas em um dia), além de características individuais 8,34,36.

Apesar de escassos, os estudos sobre as relações saúde e trabalho no grupo dos ACS evidenciaram queixas de dores nas pernas e nas costas associadas aos deslocamentos diários e aos longos percursos para acessar os domicílios dos usuários. Adicionalmente, foram descritas 
postura ortostática prolongada e inadequações do espaço e mobiliário dos domicílios aos gestos e movimentos necessários para a realização das tarefas exigidas na prestação do cuidado 37,38.

Foram observadas maiores prevalências de distúrbios musculoesqueléticos no grupo dos trabalhadores da saúde que relataram condições ambientais inadequadas. Quando comparadas às prevalências do grupo que avaliou como sendo ótimas as condições ambientais de trabalho, verificou-se gradiente positivo, sendo 33\%, 55\% e $57 \%$ maiores para o grupo que relatou condições satisfatórias, razoáveis e precárias, respectivamente. Tais resultados são convergentes aos citados na literatura, pois em estabelecimentos de saúde têm sido descritos os seguintes problemas: ruído incômodo e irritante, desconforto climático, adoção de posturas inadequadas e/ou prolongadas devido às características dos equipamentos, dos materiais e do mobiliário 39. Tais fatores podem afetar a segurança e a saúde física dos trabalhadores, assim como sua saúde mental e bem-estar 40 .

Entre os trabalhadores que executavam tarefas sob alta demanda física, a prevalência de distúrbios musculoesqueléticos foi maior quando comparada às taxas observadas no grupo que informou realizar tarefas sob baixa demanda física. Esse dado reforça os resultados obtidos em estudos nos estabelecimentos de saúde 7,24,25,27.

A etiologia dos distúrbios musculoesqueléticos é multifatorial, inclui não somente os estressores físicos, mas também fatores de risco psicossociais. Demanda psicológica e suporte social têm sido identificados como associados à ocorrência de distúrbios musculoesqueléticos entre trabalhadores da saúde ${ }^{9}$. Com relação aos fatores psicossociais, verificou-se que nenhum deles manteve significância no modelo ajustado.

\section{Limites e vantagens do estudo}

Dado o desenho transversal do estudo, não é possível estabelecer causa e efeito das associações encontradas entre o trabalho e distúrbios musculoesqueléticos.

Pesquisas baseadas em questionários respondidos e completados pelos próprios respondentes, como é o caso desta investigação, possibilitam a ocorrência de viés de autorrelato. Ainda assim, estes fornecem informações sobre a carga das doenças na população e sua utilização pode ser essencial na análise da associação da dor musculoesquelética com condições de saúde em geral 41 .

Durante a coleta, a reposição dos sorteados que não foram encontrados nos seus locais de trabalho após três tentativas respeitou a área geográfica, o nível de complexidade e o grupo ocupacional. Portanto, foram adotadas estratégias de modo a reduzir ou minimizar possíveis vieses de seleção (como a perda de trabalhadores em um ou outro grupo específico, o que poderia comprometer a representatividade da população estudada). Análise entre a composição da lista dos trabalhadores estudados e a lista completa obtida no setor de recursos humanos mostrou que o grupo estudado é comparável à população de origem, não havendo distorções significativas entre elas. Além disso, registra-se que foi estudado um número maior de trabalhadores (1.687) do que o tamanho amostral estabelecido (1.191) e que se obteve uma taxa de resposta elevada (acima de $70 \%$ ).

\section{Conclusão}

Altas prevalências de distúrbios musculoesqueléticos foram encontradas na população do estudo, com diferenciais negativos para os profissionais da odontologia e para os ACS, ambos evidentemente expostos às cargas biomecânicas. Renda e escolaridade não se mostraram associadas ao desfecho, restando como indicador de nível socioeconômico apenas a ocupação. Embora esteja reconhecido o papel dos fatores psicossociais no desencadeamento e agravamento das morbidades musculoesqueléticas, nossos achados reforçam o peso dos fatores ambientais e biomecânicos (demanda física e ocupações a ela expostas) suficientemente descritos na literatura específica. De outro lado, características individuais (sexo) e comportamentais (viver conjuntamente e atividade física) e probabilidade de TMC permaneceram significativamente associados ao desfecho. No conjunto, essas evidências confirmam a reconhecida complexidade dos distúrbios musculoesqueléticos de origem ocupacional e indicam pistas para a elaboração de programas de promoção da saúde nos estabelecimentos sanitários. 


\section{Resumo}

Estudo transversal que investigou a prevalência de distúrbios musculoesqueléticos e os fatores associados em uma amostra de 1.808 sujeitos do universo de 13.602 trabalhadores do sistema municipal de saúde de Belo Horizonte, Minas Gerais, Brasil. A prevalência foi avaliada com base no autorrelato de dor nos membros superiores, membros inferiores e dorso; a magnitude das associações foi estimada por meio da regressão de Poisson, em modelos univariados $(p \leq 0,20)$ e multivariados $(p \leq 0,05)$. A prevalência de distúrbios musculoesqueléticos foi de 49,9\%; e permaneceu positivamente associada a ser do sexo feminino; viver com um companheiro; praticar atividade física menos que duas vezes por semana; relato compatível com transtorno mental comum; exercer cargos de dentista, técnico de odontologia e agente comunitário de saúde; alta demanda física e condições de trabalho inadequadas. Os resultados confirmam a complexidade dos distúrbios musculoesqueléticos e indicam pistas para a elaboração de programas de promoção da saúde nos estabelecimentos sanitários.

Transtornos Traumáticos Cumulativos; Ocupações em Saúde; Saúde do Trabalhador; Condições de Trabalho

\section{Colaboradores}

R. E. C. Barbosa realizou a análise, interpretação dos dados e a redação final do artigo. A. A. Assunção contribuiu na concepção do estudo, coordenou o trabalho de campo e o processamento dos dados, além de contribuir na redação final do artigo. T. M. Araújo contribuiu na concepção do estudo e na redação final do artigo.

\section{Agradecimentos}

Agradecemos o financiamento da Fundação de Amparo a Pesquisa de Minas Gerais (FAPEMIG - EDT 33392006).

\section{Referências}

1. Paim JS. Recursos humanos em saúde no Brasil: problemas crônicos e desafios agudos. São Paulo: Faculdade de Saúde Pública, Universidade de São Paulo; 1994. (AdSAUDE - Série Temática, 1).

2. Secretaria de Gestão do Trabalho e da Educação na Saúde, Ministério da Saúde. Indicadores de gestão em trabalho em saúde. Brasília: Ministério da Saúde; 2007.

3. Smith DR, Leggat, PA, Araki S. Emerging occupational hazards among health care workers in the new millennium. Health 2007; 45:595-7.

4. Woods V, Buckle P. Work, inequality and musculoskeletal health. Suffolk: HSE Books; 2002. (Contract research. Report 421/2002).

5. Assunção AA, Vilela LVO. Lesões por esforços repetitivos: guia para os profissionais de saúde. Piracicaba: Centro de Referência em Saúde do Trabalhador de Piracicaba; 2009.

6. Tinubu BM, Mbada CE, Oyeyemi AL, Fabunmi AA. Work-related musculoskeletal disorders among nurses in Ibadan, South-west Nigeria: a crosssectional survey. BMC Musculoskelet Disord 2010; 11:12.
7. Henríquez MG, Rivera CF, Eyzaguirre JM. Prevalencia de trastornos músculo-esqueléticos de columna lumbar en trabajadoras y límites biomecánicos en el manejo de carga y pacientes. Cienc Trab 2010; 37:380-5.

8. Alexopoulos EC, Stathi I, Charizani F. Prevalence of musculoskeletal disorders in dentists. BMC Musculoskelet Disord 2004; 5:16.

9. Menzel NN. Psychosocial factors in musculoskeletal disorders. Crit Care Nurs Clin North Am 2007; 19:145-53.

10. Leroux I, Dionne CE, Bourbonnais R, Brisson C. Prevalence of musculoskeletal pain and associated factors in the Quebec working population. Int Arch Occup Environ Health 2005; 78:379-86.

11. Daraiseh NM, Cronin SN, Davis LS, Shell RL, Karwowski W. Low back pain symptoms among hospital nurses, associations to individual factors and pain in multiple body regions. Int J Ind Ergon 2010; 40:19-24.

12. Prefeitura de Belo Horizonte. Estrutura da Secretaria Municipal de Saúde. http://portalpbh.pbh. gov.br (acessado em 21/Nov/2010). 
13. Stendig-Lindberg G. Prevalences of musculoskeletal pain and its socio-demographic correlates in an urban Israeli population sample. Eur J Phys Med Rehabil Med 1998; 8:77-80.

14. Brandão AG, Horta BL, Tomasi E. Sintomas de distúrbios osteomusculares em bancários de Pelotas e região: prevalência e fatores associados. Rev Bras Epidemiol 2005; 8:295-305.

15. Cardoso JP, Araújo TM, Carvalho FM, Oliveira NF, Reis EJFB. Aspectos psicossociais do trabalho e dor musculoesquelética em professores. Cad Saúde Pública 2011; 27:1498-506.

16. World Health Organization. Global atlas of the health workforce. Geneva: World Health Organization; 2009.

17. Santos KOB, Araújo TM, Oliveira NF. Estrutura fatorial e consistência interna do Self-Reporting Questionnaire (SRQ-20) em população urbana. Cad Saúde Pública 2009; 25:214-22.

18. Araújo TM, Aquino E, Menezes G, Santos CO, Aguiar L. Aspectos psicossociais do trabalho e distúrbios psíquicos entre trabalhadoras de enfermagem. Rev Saúde Pública 2003; 37:424-33.

19. Araújo TM, Pinho PS, Almeida MM. Prevalência de transtornos mentais comuns em mulheres e sua relação com as características sociodemográficas e o trabalho doméstico. Rev Bras Saúde Matern Infant 2005; 5:337-48.

20. Thierney D, Romito P, Messing K. She ate not the bread of idleness: exhaustion is related to domestic and salaried working conditions among 539 Québec hospital workers. Women Health 1990; 16:21-42.

21. Karasek RA, Theörell T. Healthy work-stress, productivity, and the reconstruction of working life. New York: Basic Books; 1990.

22. Araújo TM, Graça CC, Araújo EA. Estresse ocupacional e saúde: contribuições do modelo demanda-controle. Ciênc Saúde Coletiva 2003; 8:9911003.

23. Souza SF, Carvalho FM, Araújo TM, Porto LA. Fatores psicossociais do trabalho e transtornos mentais comuns em eletricitários. Rev Saúde Pública 2010; 44:710-7.

24. Monteiro MS, Alexandre NM, Rodrigues CM. Doenças musculoesqueléticas, trabalho e estilo de vida entre trabalhadores de uma instituição pública de saúde. Rev Esc Enferm USP 2006; 40:20-5.

25. Alamgir H, Cvitkovich Y, Yu S, Yassi A. Work-related injury among direct care occupations in British Columbia, Canada. Occup Environ Med 2007; 64:769-75.

26. Picavet HS, Schouten JS. Musculoskeletal pain in the Netherlands: prevalences, consequences and risk groups, the DMC3-study. Pain 2003; 102: 167-78.

27. Ngan K, Drebit S, Siow S, Yu S, Keen D, Alamgir H. Risks and causes of musculoskeletal injuries among health care workers. Occup Med (Lond) 2010; 60:389-94.
28. Strazdins L, Bammer G. Women, work and musculoskeletal health. Soc Sci Med 2004; 58:997-1005.

29. Garcia LP, Höfelmann DA, Facchini LA. Self-rated health and working conditions among workers from primary health care centers in Brazil. Cad Saúde Pública 2010; 26:971-80.

30. Verbunt JA, Sieben JM, Seelen HA, Vlaeyen JW, Bousema EJ, Heijden GJ, et al. Decline in physical activity, disability and pain-related fear in subacute low back pain. Eur J Pain 2005; 9:417-25.

31. Hurwitz EL, Morgenstern H, Chiao C. Effects of recreational physical activity and back exercises on low back pain and psychological distress: findings from the UCLA low back pain study. Am J Public Health 2005; 95:1817-24.

32. Mello MT, Boscolo RA, Esteves AM, Tufik S. O exercício físico e os aspectos psicobiológicos. Rev Bras Med Esporte 2005; 11:203-7.

33. Guimarães JM, Caldas CP. A influência da atividade física nos quadros depressivos de pessoas idosas: uma revisão sistemática. Rev Bras Epidemiol 2006; 9:481-92.

34. Santos Filho SB, Barreto SM. Atividade ocupacional e prevalência de dor osteomuscular em cirurgiões-dentistas de Belo Horizonte, Minas Gerais, Brasil: contribuição ao debate sobre os distúrbios osteomusculares relacionados ao trabalho. Cad Saúde Pública 2001; 17:181-93.

35. Passier L, McPhail S. Work related musculoskeletal disorders amongst therapists in physically demanding roles: qualitative analysis of risk factors and strategies for prevention. BMC Musculoskelet Disord 2011; 12:24.

36. Leggat PA, Smith DR. Musculoskeletal disorders self-reported by dentists in Queensland, Australia. Aust Dent J 2006; 51:324-7.

37. Trindade LL, Gonzales RM, Beck CL, Lautert L. Cargas de trabalho entre os agentes comunitários de saúde. Rev Gaúch Enferm 2007; 28:473-9.

38. Nascimento GM, David HM. Avaliação de riscos no trabalho dos agentes comunitários de saúde: um processo participativo. Rev Enferm UERJ 2008; $16: 550-6$.

39. Chiodi MB, Marziale MH. Riscos ocupacionais para trabalhadores de UBS: revisão bibliográfica. Acta Paul Enferm 2006; 19:212-7.

40. Organización Panamericana de la Salud/Organización Mundial de la Salud. Ambientes de trabajo saludables: un modelo para la acción para empleadores, trabajadores, autoridades normativas y profesionales. Geneva: Organización Mundial de la Salud; 2010.

41. Picavet HS, Hazes JM. Prevalence of self reported musculoskeletal diseases is high. Ann Rheum Dis 2003; 62:644-50.

Recebido em 18/Out/2011

Versão final reapresentada em 09/Mai/2012 Aprovado em 18/Mai/2012 